\title{
Afatinib versus gemcitabine/cisplatin for first-line treatment of Chinese patients with advanced non-small-cell lung cancer harboring EGFR mutations: subgroup analysis of the LUX-Lung 6 trial
}

This article was published in the following Dove Press journal: OncoTargets and Therapy

Yi-Long Wu,' Chong-Rui Xu,' Cheng-Ping $\mathrm{Hu}{ }^{2}$ Jifeng Feng, ${ }^{3}$ Shun Lu, ${ }^{4}$ Yunchao Huang, ${ }^{5}$ Wei $\mathrm{Li}^{6}{ }^{6}$ Mei Hou, ${ }^{7}$ Jian Hua Shi, ${ }^{8}$ Angela Märten, ${ }^{9}$ Jean Fan, ${ }^{10}$ Barbara Peil," Caicun Zhou ${ }^{12}$

'Guangdong Lung Cancer Institute, Guangdong General Hospital and Guangdong Academy of Medical Sciences, Guangzhou, Guangdong, China; ${ }^{2}$ Department of Pulmonary Medicine. Xiangya Hospital, Central South University, Changsha, Hunan, China; ${ }^{3}$ Department of Internal Medicine, Jiangsu Provincial Tumor Hospital, Nanjing, Jiangsu, China; ${ }^{4}$ Shanghai Lung Cancer Center, Shanghai Chest Hospital, Shanghai Jiao Tong University, Shanghai, China; ${ }^{5}$ Department of Thoracic Surgery, Yunnan Tumor Hospital (The Third Affiliated Hospital of Kunming Medical University), Kunming, Yunnan, China; ${ }^{6}$ Department of Hematology \& Oncology, Cancer Center, First Hospital of Jilin University, Changchun, Jilin, China; ${ }^{7}$ Lung Cancer Centre, West China Hospital, Sichuan University, Chengdu, Sichuan, China; ${ }^{8}$ Department of Oncology, Lin Yi Tumor Hospital, Linyi, Shandong, China; 'Department of Oncology, Boehringer Ingelheim $\mathrm{GmbH}$, Ingelheim, Germany; ${ }^{10}$ Department of Oncology, Boehringer Ingelheim Pharmaceuticals, Inc., Ridgefield, CT, USA; "Department of Oncology, Boehringer Ingelheim Pharma GmbH \& Co. KG, Ingelheim, Germany; ${ }^{12}$ Department of Medical Oncology, Shanghai Pulmonary Hospital, Yangpu District, Shanghai, China

Correspondence: Yi-Long Wu

Guangdong Lung Cancer Institute, Guangdong General Hospital and Guangdong Academy

of Medical Sciences, 106 Zhongshan Er Road,

Guangzhou 510080, Guangdong, China

Tel +86 2083877855

Fax +86 2083827712

Email syylwu@live.cn
Introduction: Non-small-cell lung cancer (NSCLC) is the leading cause of cancer death in China. Four epidermal growth factor receptor (EGFR)-targeted tyrosine kinase inhibitors afatinib, erlotinib, icotinib, and gefitinib - are available for first-line treatment of NSCLC in China; however, there are few data to guide treatment choice. The Phase III LUX-Lung 6 trial compared afatinib with platinum-based chemotherapy for first-line treatment of patients from Southeast Asia with EGFR mutation-positive advanced NSCLC. This post hoc analysis assessed the findings from LUX-Lung 6 in Chinese patients.

Clinical trial registration: ClinicalTrials.gov: NCT01121393.

Materials and methods: Previously untreated patients with EGFR mutation-positive stage IIIB/ IV lung adenocarcinoma were randomized 2:1 to receive afatinib or $\leq 6$ cycles of gemcitabine/ cisplatin. The key outcomes were progression-free survival (PFS; primary), objective response rate, disease control rate, overall survival (OS), duration of response and disease control, patientreported outcomes, and safety. Three hundred and twenty-seven patients from mainland China were treated (89.8\% of overall LUX-Lung 6 population; afatinib 217, gemcitabine/cisplatin 110).

Results: PFS was significantly longer with afatinib than gemcitabine/cisplatin (median 11.0 versus 5.6 months; hazard ratio [HR], 0.30 [95\% CI, 0.21, 0.43]; $P<0.0001$ ). Overall, there was no significant difference in OS between treatment arms; however, in a subgroup analysis, afatinib significantly improved OS versus gemcitabine/cisplatin in patients with an EGFR Del19 mutation (median 31.6 versus 16.3 months; HR, 0.61 [95\% CI, 0.41, 0.91]; $P=0.0146$ ). Afatinib was well tolerated, with most treatment-related adverse events (TRAEs) being of grade 1 or 2 severity. The most common grade 3/4 TRAEs with afatinib were rash/acne (15.9\%/0.5\%), stomatitis $(6.1 \% / 0 \%)$, and diarrhea $(5.6 \% / 0 \%)$. TRAEs leading to permanent discontinuation were reported in 12 patients $(5.6 \%)$ receiving afatinib and $43(41.7 \%)$ receiving gemcitabine/cisplatin. Afatinib significantly improved PFS compared with standard first-line chemotherapy in Chinese patients with EGFR mutation-positive NSCLC and demonstrated a manageable safety profile.

Conclusion: The findings support the rationale for using afatinib as a first-line treatment option for this patient population.

Keywords: afatinib, NSCLC, EGFR, first-line, Phase III, Chinese patients

\section{Introduction}

In China, lung cancer is the most predominant malignancy and the leading cause of cancer death. ${ }^{1}$ Furthermore, its incidence and mortality are rapidly increasing; the estimated number of deaths due to lung cancer in China rose from 493,348 in 2008 
to 529,153 in $2011 .{ }^{2}$ Unfortunately, the majority of patients with lung cancer in China are diagnosed at an advanced stage, thus precluding the possibility of surgery with curative intent. ${ }^{2,3}$ The mainstay of treatment for advanced-stage disease is chemotherapy; however, the effectiveness of systemic platinum-doublet chemotherapy has plateaued over recent years and remains suboptimal. Typically, median survival is only $12-13$ months. ${ }^{4}$ Therefore, there remains a high unmet need for further treatment options in this setting, particularly in China.

Globally, the epidermal growth factor receptor (EGFR) tyrosine kinase inhibitors (TKIs), erlotinib and gefitinib, and the irreversible ErbB family blocker, afatinib, are widely used as a first-line treatment for patients with EGFR mutationpositive non-small-cell lung cancer (NSCLC). ${ }^{5}$ In China, an additional first-generation EGFR TKI, icotinib, is also available. ${ }^{6}$ In Phase III trials, all of these agents significantly improved progression-free survival (PFS) versus platinumdoublet chemotherapy in this treatment setting, and conferred predictable and manageable tolerability profiles consistent with inhibition of EGFR signaling. ${ }^{7-15}$ Recent data, however, suggest that afatinib may offer more favorable clinical outcomes than the first-generation reversible EGFR TKIs, possibly reflecting its broader inhibitory profile. ${ }^{16-18}$ In two Phase III trials - LUX-Lung 3 (global) and LUX-Lung 6 (China, South Korea, and Thailand) - prespecified analyses have demonstrated individually that afatinib conferred superior overall survival (OS) versus standard platinum-doublet chemotherapy in treatment-naïve patients whose tumors harbor a Del19 mutation, the most common type of mutation observed in EGFR. ${ }^{16}$ To our knowledge, neither gefitinib and icotinib nor erlotinib have ever demonstrated an OS advantage over chemotherapy for any patient group. Furthermore, in a recent Phase IIb study (LUX-Lung 7), first-line afatinib significantly improved PFS, time-to-treatment failure (TTF), and objective response rate (ORR) compared with gefitinib in patients with EGFR mutation-positive NSCLC. ${ }^{17,18}$ Afatinib also demonstrated a trend for longer OS. ${ }^{17,18}$

Afatinib is associated with a similar safety profile to firstgeneration EGFR TKIs, although certain treatment-related grade $\geq 3$ adverse events (AEs) occur more frequently with different agents. In LUX-Lung 7, for instance, diarrhea ( $13 \%$ versus $1 \%$ ), rash/acne ( $9 \%$ versus $3 \%$ ), and fatigue (6\% versus $0 \%$ ) were all higher with afatinib than with gefitinib. In contrast, other grade $\geq 3 \mathrm{AEs}$, notably elevated liver enzymes ( $0 \%$ versus $9 \%$ ) and interstitial lung disease $(0 \%$ versus $2 \%$ ), were less common with afatinib than gefitinib. ${ }^{17}$
One patient in the gefitinib arm died from drug-related hepatic and renal failure, while no drug-related fatal events occurred in the afatinib arm. Afatinib is available in multiple dose-strength formulations, thus facilitating tolerability-guided dose adjustments. This approach renders the majority of treatment-related AEs (TRAEs) manageable, reducing the need for treatment discontinuation. In LUX-Lung 7, for example, treatment discontinuations were equally low with afatinib as they were with gefitinib ( $6 \%$ each) ${ }^{17}$ Importantly, recent data indicated that the efficacy of afatinib treatment is not compromised in those patients who require dose reduction. ${ }^{19}$ Based on these efficacy and safety observations, there is rationale for considering afatinib as a first-line treatment option in patients with EGFR mutation-positive NSCLC.

In China, choice of EGFR TKI is particularly important, given the elevated frequency of EGFR mutations in this population compared with others. Notably, the frequency of EGFR mutations is $5 \%-10 \%$ in Caucasians, ${ }^{20-22}$ but is accepted to be $\sim 50 \%$ in Asian and Chinese patients with adenocarcinoma. ${ }^{21}$ Among Chinese patients, Del19 was the most common mutation $(-50 \%)$ followed by L858R. ${ }^{23}$

The LUX-Lung 6 study compared afatinib with gemcitabine/cisplatin for first-line treatment of patients from Southeast Asia with EGFR mutation-positive advanced NSCLC. In practice, however, most of the patients in LUX-Lung 6 came from China, providing an opportunity to examine outcomes with afatinib specifically in this population. This post hoc analysis was performed to assess the efficacy and safety of afatinib relative to gemcitabine/cisplatin in the subgroup of Chinese patients participating in LUX-Lung 6.

\section{Materials and methods Patients and study design}

The study design and eligibility criteria of LUX-Lung 6 (NCT01121393) have been published previously. ${ }^{12}$ In brief, LUX-Lung 6 was a randomized, open-label, Phase III study conducted in China, Thailand, and South Korea (Supplementary slides). Of the 36 centers participating in the study, 30 were in China. Eligible patients had pathologically confirmed previously untreated stage IIIB (with pleural effusion) or IV lung adenocarcinoma, an Eastern Cooperative Oncology Group (ECOG) performance status (PS) of 0 or 1, measurable disease according to Response Evaluation Criteria in Solid Tumors, version 1.1, and adequate organ function. In addition, tumor tissue had to be EGFR mutation-positive as assessed at a central laboratory using the Therascreen EGFR 29 kit (Qiagen, Manchester, UK). Eligible patients 
were randomly assigned to receive afatinib or gemcitabine/ cisplatin in a 2:1 ratio, stratified by EGFR mutation (L858R, Del19, or other).

The study was conducted in accordance with the Declaration of Helsinki and guidelines on Good Clinical Practice, and the protocol was approved by local ethics committees at each participating center (Table S1). All patients provided written informed consent for trial participation.

\section{Treatments}

Patients received either oral afatinib (40 $\mathrm{mg} /$ day) or up to 6 cycles of cisplatin $75 \mathrm{mg} / \mathrm{m}^{2}$ on day 1 plus gemcitabine $1,000 \mathrm{mg} / \mathrm{m}^{2}$ on days 1 and 8 of a 21-day cycle. Patients continued treatment until disease progression, intolerable toxic effects, or withdrawal of consent. Patients receiving afatinib were permitted to dose escalate to $50 \mathrm{mg}$ /day after the first 21 -day cycle in the absence of grade $>1$ TRAEs. Afatinib was withheld for up to 14 days and then dose reduced by $10 \mathrm{mg}$ decrements (minimum $20 \mathrm{mg} /$ day) for treatment-related grade 3 or selected prolonged grade 2 AEs. Dose reductions or interruptions for patients receiving gemcitabine/cisplatin were in accordance with guidance provided in the current summaries of product characteristics. Patients who remained on afatinib treatment for $\geq 36$ months were defined as longterm responders (LTRs).

\section{Outcomes and assessments}

The primary end point was PFS according to independent central review. Key secondary end points were ORR (complete response $[\mathrm{CR}]$ or partial response $[\mathrm{PR}]$ ), disease control rate (DCR; CR, PR, or stable disease), OS, duration of response and disease control, patient-reported outcomes (PROs), and safety.

Tumor assessments were performed by computed tomography or magnetic resonance imaging every 6 weeks for the first 48 weeks and then every 12 weeks thereafter until disease progression or start of new anticancer therapy. Brain imaging and bone scans were performed if clinically indicated. Scans were reviewed by an independent central imaging review group consisting of radiologists and oncologists.

PROs were assessed at randomization and every 3 weeks thereafter until disease progression or start of new anticancer therapy using the self-administered cancer-specific European Organisation for Research and Treatment of Cancer quality of life core questionnaire QLQ-C30, ${ }^{24}$ and the lung cancerspecific module QLQ-LC13. ${ }^{25}$ Safety was monitored weekly, with incidence and intensity of AEs graded according to
National Cancer Institute Common Terminology Criteria for Adverse Events version 3.0.

\section{Pharmacokinetics}

For patients randomized to the afatinib arm, blood sampling was performed to estimate the afatinib trough (predose) plasma concentrations at steady state. Plasma afatinib concentrations (free base) were analyzed using a validated high-performance liquid chromatography tandem mass spectrometry method at Boehringer Ingelheim Pharma GmbH \& Co. KG, Drug Metabolism and Pharmacokinetics Germany, Biberach, Germany.

\section{Statistical analyses}

Kaplan-Meier estimates were used to construct survival curves and calculate median PFS and OS. A Cox proportionalhazard model was used to derive hazard ratios (HRs) and 95\% confidence intervals (CIs); treatment groups were compared using a log-rank test. Prespecified subgroup analyses were conducted by sex, age ( $<65$ years versus $\geq 65$ years), EGFR mutation type (Del19 versus L858R versus other), ECOG PS (0 versus 1), and smoking history.

PROs focused on the NSCLC-related symptoms of cough (question 1 of QLQ-LC13), dyspnea (questions 3-5 of QLQ-LC13 and question 8 of QLQ-C30), and pain (questions 9 and 19 of QLQ-C30, and questions 10-12 of QLQ-LC13). The following analyses were undertaken: percentage of patients whose symptoms improved ( $\geq 10$-point decrease from baseline score), remained stable, or deteriorated ( $\geq 10$-point increase from baseline score); time to deterioration of symptoms; and mean difference in symptom scores over time (longitudinal analysis). Statistical analyses were performed using SAS (version 9.2 or later).

\section{Results}

\section{Patients and baseline characteristics}

Of the 364 patients randomized in LUX-Lung 6,327 (89.8\%) were from mainland China. A total of 217 patients were randomized to afatinib; 110 patients were randomized to gemcitabine/cisplatin. Three patients in the afatinib group and seven in the gemcitabine/cisplatin group did not receive any treatment; thus, the treated sets comprised 214 and 103 patients, respectively.

There were no notable differences in baseline characteristics between the Chinese subgroup and the overall LUX-Lung 6 population (Table 1). Consistent with the overall LUX-Lung 6 population, there was a slightly higher 
Table I Patient demographic and baseline characteristics in the overall LUX-Lung 6 population and the Chinese subgroup

\begin{tabular}{|c|c|c|c|c|}
\hline \multirow[t]{2}{*}{ Characteristic } & \multicolumn{2}{|c|}{ Overall population $(\mathrm{N}=364)$} & \multicolumn{2}{|c|}{ Chinese patients $(\mathrm{N}=327)$} \\
\hline & $\begin{array}{l}\text { Afatinib } \\
(n=242)\end{array}$ & $\begin{array}{l}\text { Gem/Cis } \\
(n=122)\end{array}$ & $\begin{array}{l}\text { Afatinib } \\
(n=2 \mid 7)\end{array}$ & $\begin{array}{l}\text { Gem/Cis } \\
(n=I \mid 0)\end{array}$ \\
\hline \multicolumn{5}{|l|}{ Sex, n (\%) } \\
\hline Female & $155(64.0)$ & $83(68.0)$ & $136(62.7)$ & $76(69.1)$ \\
\hline Male & $87(36.0)$ & $39(32.0)$ & $81(37.3)$ & $34(30.9)$ \\
\hline Median age, years (range) & $58(29-79)$ & $58(27-76)$ & $58(30-78)$ & $58(27-75)$ \\
\hline \multicolumn{5}{|l|}{ Race/ethnicity, ${ }^{\mathrm{n}}(\%)$} \\
\hline Southeast Asian & $14(5.8)$ & $10(8.2)$ & 0 & 0 \\
\hline South Korean & II (4.5) & $2(1.6)$ & 0 & 0 \\
\hline Chinese & $217(89.7)$ & I IO (90.2) & $217(100)$ & $110(100)$ \\
\hline \multicolumn{5}{|l|}{ ECOG performance status, $n(\%)$} \\
\hline 0 & $48(19.8)$ & $4 I(33.6)$ & $44(20.3)$ & $40(36.4)$ \\
\hline I & $194(80.2)$ & $81(66.4)$ & $173(79.7)$ & $70(63.6)$ \\
\hline \multicolumn{5}{|l|}{ Smoking status, n (\%) } \\
\hline Never smoked & I8I (74.8) & $99(8 I .1)$ & $159(73.3)$ & $92(83.6)$ \\
\hline Other current or ex-smoker & $53(21.9)$ & $19(15.6)$ & $50(23.0)$ & $15(13.6)$ \\
\hline$<15$ pack-years and stopped $>1$ year ago & $8(3.3)$ & $4(3.3)$ & $8(3.7)$ & $3(2.7)$ \\
\hline \multicolumn{5}{|l|}{ Adenocarcinoma stage, $\mathrm{n}(\%)$} \\
\hline IIIB & $16(6.6)$ & $6(4.9)$ & $13(6.0)$ & $6(5.5)$ \\
\hline IV & $226(93.4)$ & $116(95.1)$ & $204(94.0)$ & $104(94.5)$ \\
\hline \multicolumn{5}{|l|}{ Number of metastatic sites, $\mathrm{n}(\%)$} \\
\hline 0 & $5(2.1)$ & $\mathrm{I}(0.8)$ & $3(1.4)$ & $\mathrm{I}(0.9)$ \\
\hline I & $72(29.8)$ & $52(42.6)$ & $65(30.0)$ & $48(43.6)$ \\
\hline 2 & $91(37.6)$ & $36(29.5)$ & $84(38.7)$ & $30(27.3)$ \\
\hline$\geq 3$ & $74(30.6)$ & $33(27.0)$ & $65(30.0)$ & $31(28.2)$ \\
\hline \multicolumn{5}{|l|}{ EGFR mutation, $\mathrm{n}(\%)$} \\
\hline Common mutations & $216(89.3)$ & $108(88.5)$ & $193(88.9)$ & 97 (88.2) \\
\hline Dell9 & $124(5 \mid .2)$ & $62(50.8)$ & $\mathrm{II} \mid(5 \mathrm{I} .2)$ & $55(50.0)$ \\
\hline L858R & $92(38.0)^{\mathrm{b}}$ & $46(37.7)$ & $82(37.8)^{b}$ & $42(38.2)$ \\
\hline Uncommon mutations ${ }^{c}$ & $26(10.7)$ & $14(\mid \mathrm{I} .5)$ & $24(I I . I)$ & $13(\mid 1.8)$ \\
\hline
\end{tabular}

Notes: ${ }^{a}$ Available categories within Asian race included Indian subcontinent Asian, Southeast Asian, Japanese, Korean, Taiwanese or Chinese, Asian - other. ${ }^{\natural}$ Including four patients with both L858R and Dell 9 mutation. Including T790M, exon 20 insertions, G7I9X, S768I, and L86IQ, alone or as complex mutations in two or more exons. Abbreviations: Cis, cisplatin; ECOG, Eastern Cooperative Oncology Group; EGFR, epidermal growth factor receptor; Gem, gemcitabine.

proportion of males $(37.3 \%$ versus $30.9 \%$ ), slightly more patients with ECOG PS 1 (79.7\% versus $63.6 \%)$, slightly more current or ex-smokers (23.0\% versus $13.6 \%)$, and more patients with two metastatic sites (38.7\% versus $27.3 \%$ ) in the afatinib group compared to the gemcitabine/cisplatin group (Supplementary slides).

\section{Treatment exposure}

Median treatment duration with afatinib was 389.5 days (range 3-1, 256 days); 37 patients had their afatinib dose escalated to $50 \mathrm{mg} /$ day. At the end of treatment, 141 patients (65.9\%) had had no dose reductions, 59 (27.6\%) had one dose reduction, and 14 (6.5\%) had two dose reductions. The median time to first dose reduction with afatinib was 72 days and the median dose interruption before dose reduction was 6 days. Median duration of treatment on gemcitabine/ cisplatin was 86 days (range 1-154 days). A total of 36 patients (38.7\%) received all of their gemcitabine/cisplatin courses without interruption; 26 (28.0\%) had a worst delay of 4-6 days and $31(33.3 \%)$ had a worst delay of $>6$ days. At the time of analysis, there were 20 Chinese afatinib LTRs, with a median treatment duration of 56.8 months (range: 37.3-68.2 months); eight patients remained on treatment. Dose modifications among the LTRs are shown in Figure 1 and do not indicate an impact of dose adjustment on longterm responses (Supplementary slides).

\section{PFS}

Independently assessed median PFS was 11.0 months with afatinib versus 5.6 months with gemcitabine/cisplatin (HR, 0.30 [95\% CI, 0.21, 0.43]; $P<0.0001$; Figure 2A). Significant PFS advantage with afatinib over gemcitabine/ cisplatin was also observed in patients with common $E G F R$ (Del19 or L858R) mutations (median 11.0 versus 5.6 months; HR, 0.26 [95\% CI, 0.18, 0.37]; $P<0.0001)$. Similar PFS advantage with afatinib over gemcitabine/cisplatin was observed in other patient subgroups, including individual mutation categories (Figure 2B; Supplementary slides); PFS 


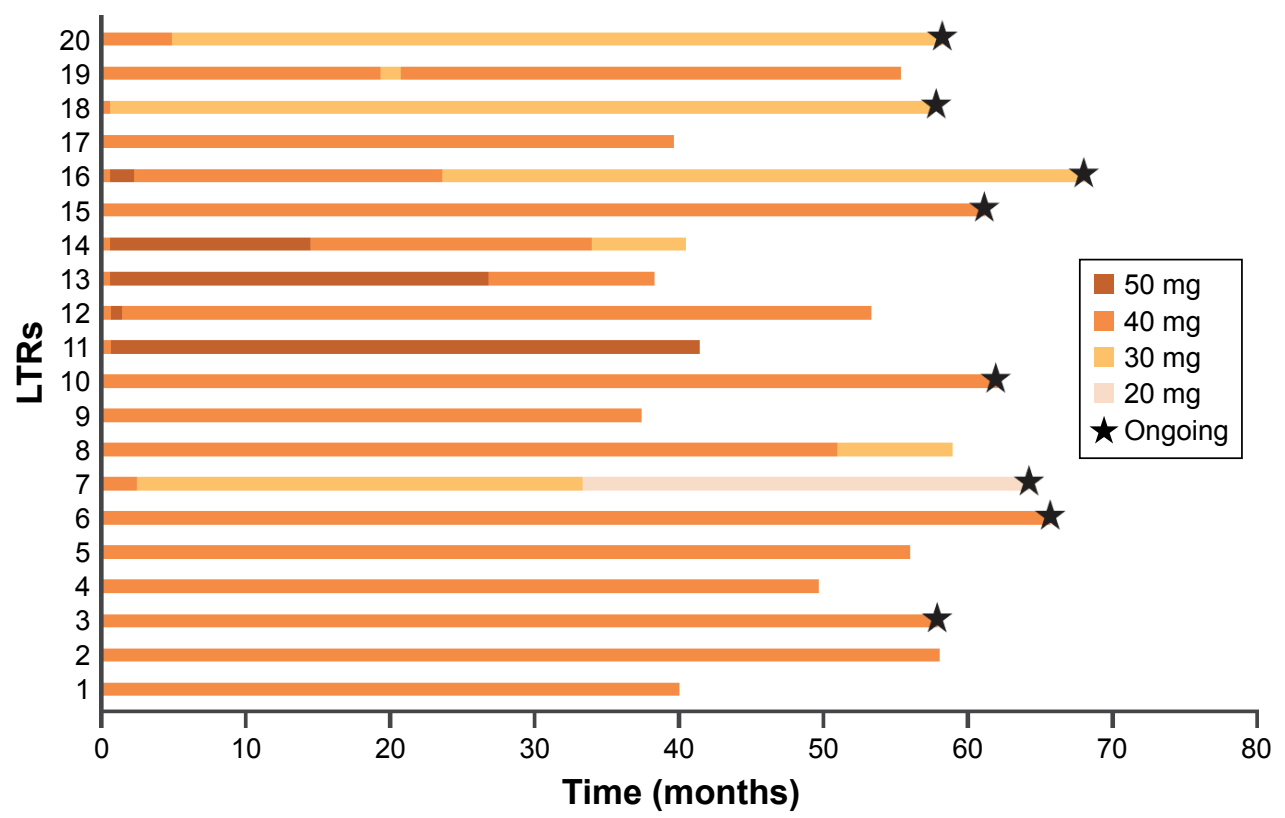

Figure I Dose adjustments among Chinese patients who were LTRs to afatinib. Abbreviation: LTRs, long-term responders.

was significantly prolonged with afatinib versus gemcitabine/ cisplatin both in patients with an EGFR Del19 mutation (median 13.7 versus 5.6 months with gemcitabine/cisplatin; HR, 0.21 [95\% CI, 0.13, 0.36]; $P<0.0001$ ) and those with the $E G F R$ L858R mutation (median 8.3 versus 5.6 months; HR, 0.33 [95\% CI, 0.20, 0.56]; $P<0.0001)$.

\section{OS}

Overall, in Chinese patients, OS was not significantly different with afatinib and gemcitabine/cisplatin (median 23.1 versus 23.2 months; HR, 0.96 [95\% CI, $0.73,1.27$ ]; $P=0.7765$; Figure $3 \mathrm{~A}$ ); median OS in patients with common mutations was 23.6 versus 23.0 months, respectively (HR, 0.82 [95\% CI, 0.62, 1.10]; $P=0.1917)$. However, in patients with an EGFR Del19 mutation, afatinib significantly improved OS versus gemcitabine/cisplatin (median 31.6 versus 16.3 months; HR, 0.61 [95\% CI, 0.41, 0.91]; $P=0.0146$; Figure $3 \mathrm{~B}$ ); there was no significant difference in the EGFR L858R subgroup (median 18.7 versus 24.5 months; HR, 1.31 [95\% CI, 0.85, 1.99]; $P=0.2171$; Figure 3C). Among the 20 patients with long-term response on afatinib (LTRs), five patients had survival times of more than 5 years (Supplementary slides).

\section{Tumor response}

The ORR was significantly higher with afatinib than with gemcitabine/cisplatin ( $66.8 \%$ versus $23.6 \%$; odds ratio [OR], 6.94 [95\% CI, 4.05, 11.88]; $P<0.0001)$; three patients $(1.4 \%)$ experienced a CR with afatinib (versus none with gemcitabine/ cisplatin; Table 2). The DCR was also significantly higher with afatinib than with gemcitabine/cisplatin $(92.2 \%$ versus 77.3\%; OR, 3.45 [95\% CI, 1.77, 6.71]; $P=0.0003$ ).

Most patients who achieved an objective response with afatinib did so within the first 6 weeks $(103 / 145,71 \%)$, that is, by the first visit, and almost all of those who achieved an objective response did so by 18 weeks (137/145, 94.5\%). The median duration of objective response was 9.7 months with afatinib and 4.2 months with gemcitabine/cisplatin. The median duration of disease control was 11.1 months with afatinib and 5.6 months with gemcitabine/cisplatin.

Significantly more patients achieved an objective response with afatinib compared with gemcitabine/ cisplatin in all of the mutation subgroups examined (Table 2). The difference between the treatment arms was particularly pronounced in patients with a Del19 mutation (77.5\% versus $27.3 \%$; OR, 9.17 [95\% CI, 4.37, 19.26]; $P<0.0001)$. The DCR was only significantly different between the treatment groups in patients with a Del19 mutation (93.7\% versus $76.4 \%$; OR, 4.60 [95\% CI, 1.72, 12.33]; $P=0.0024)$.

\section{Safety}

The most common TRAEs are shown in Table 3. Almost all patients experienced at least one TRAE (98.6\%-99.0\%). Most TRAEs were of grade 1 or 2 severity. Similar proportions of patients experienced grade 3 TRAEs in each treatment arm $35.5 \%$ and $35.9 \%$ with afatinib and chemotherapy, 
A

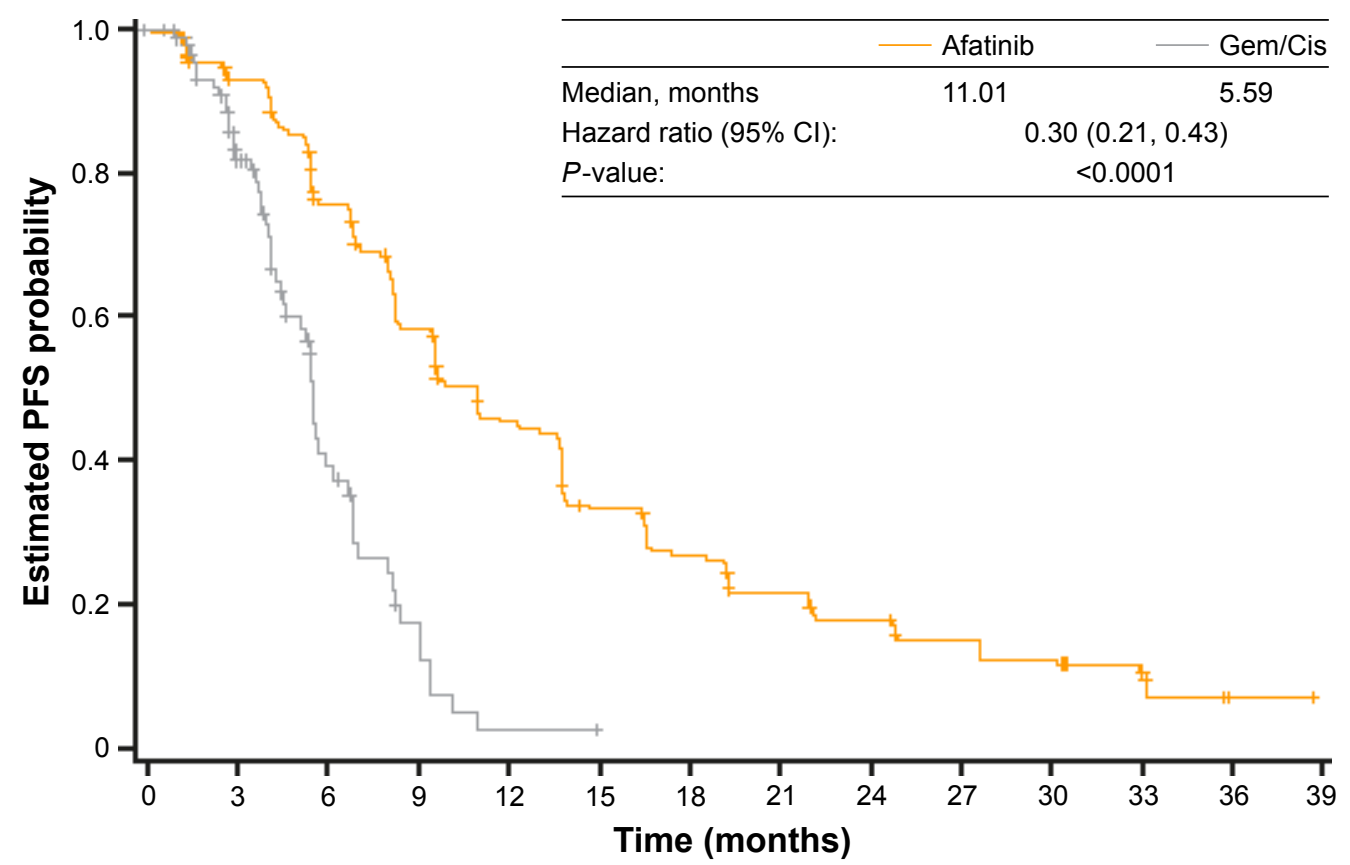

Number at risk

$\begin{array}{rllllllllllllll}\text { Afatinib } & 217 & 185 & 146 & 110 & 82 & 58 & 46 & 35 & 28 & 22 & 18 & 10 & 1 & 0 \\ \text { Gem/Cis } & 110 & 62 & 21 & 7 & 1 & 0 & 0 & 0 & 0 & 0 & 0 & 0 & 0 & 0\end{array}$

B

\begin{tabular}{|c|c|c|c|c|}
\hline & $\begin{array}{l}\text { Number of } \\
\text { patients }\end{array}$ & & & $\begin{array}{l}\text { Hazard } \\
\text { ratio }\end{array}$ \\
\hline Total & 327 & $\mapsto-1$ & & 0.301 \\
\hline \multicolumn{5}{|l|}{ Gender } \\
\hline Male & 115 & $\longmapsto$ & & 0.375 \\
\hline Female & 212 & $\longmapsto$ & & 0.251 \\
\hline \multicolumn{5}{|l|}{ Age at baseline $(<65$ versus $\geq 65)$} \\
\hline$<65$ years & 256 & $\longmapsto$ & & 0.305 \\
\hline$\geq 65$ years & 71 & $\longrightarrow$ & & 0.213 \\
\hline \multicolumn{5}{|l|}{ EGFR mutation category } \\
\hline Del19/L858R (common) & 290 & $\longmapsto-1$ & & 0.259 \\
\hline Del19 & 166 & $\longmapsto$ & & 0.213 \\
\hline L858R & 124 & $\longmapsto$ & & 0.332 \\
\hline Other (uncommon) & 37 & $\longmapsto$ & $\longrightarrow$ & 0.651 \\
\hline \multicolumn{5}{|l|}{ Baseline ECOG score } \\
\hline 0 & 84 & $\longmapsto$ & & 0.222 \\
\hline 1 & 243 & $\longmapsto$ & & 0.312 \\
\hline \multicolumn{5}{|l|}{ Smoking history } \\
\hline Never smoked & 251 & $\longmapsto-1$ & & 0.256 \\
\hline$<15$ pack year+stop $>1$ year & 11 & & & 0.189 \\
\hline \multirow[t]{2}{*}{ Oth current/ex-smoker } & 65 & $\longmapsto$ & -1 & 0.513 \\
\hline & & $\stackrel{{ }_{1 / 16}}{ }$ & $\begin{array}{l}1 \\
4\end{array}$ & $7_{16}$ \\
\hline
\end{tabular}

Figure 2 (A) PFS in Chinese patients receiving afatinib and gemcitabine/cisplatin (independent review) and (B) forest plot of PFS for subgroups of special interest (independent review).

Abbreviations: Cis, cisplatin; ECOG, Eastern Cooperative Oncology Group; EGFR, epidermal growth factor receptor; Gem, gemcitabine; PFS, progression-free survival. 

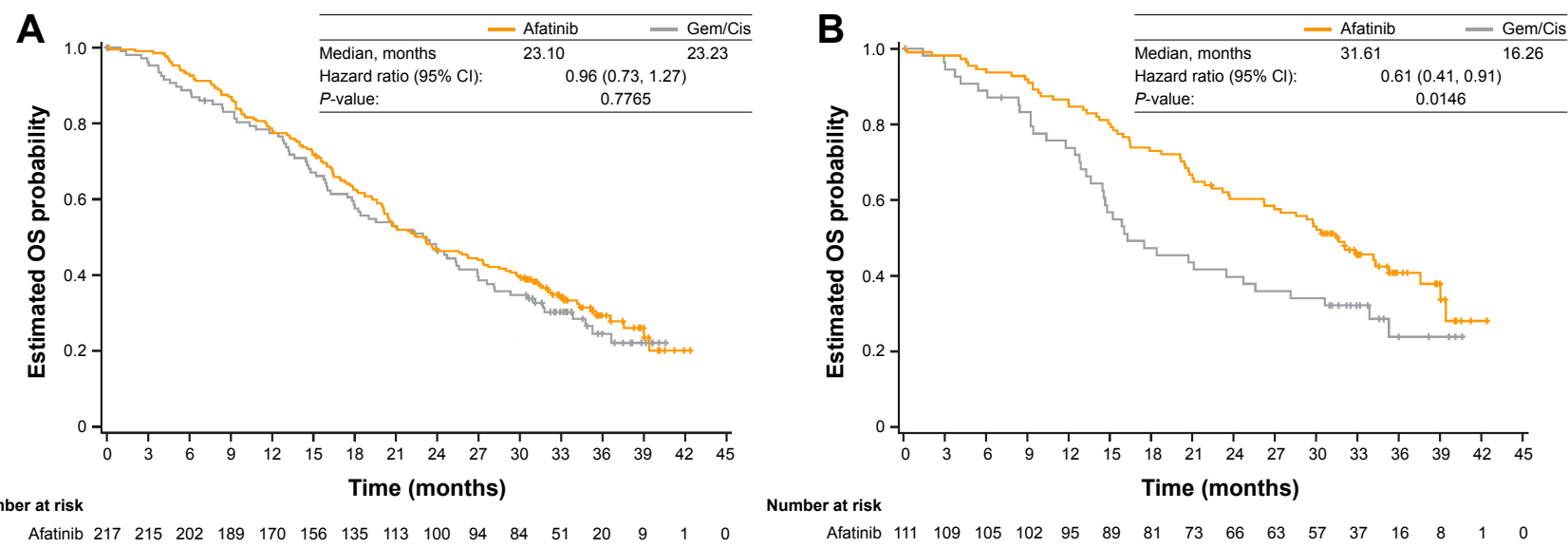

$\begin{array}{rllllllllllllllll}\text { Afatinib } & 217 & 215 & 202 & 189 & 170 & 156 & 135 & 113 & 100 & 94 & 84 & 51 & 20 & 9 & 1 & 0 \\ \text { Gem/Cis } & 110 & 103 & 95 & 88 & 82 & 71 & 62 & 56 & 49 & 41 & 36 & 21 & 10 & 4 & 0 & 0\end{array}$

Number at risk

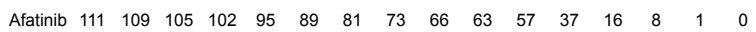
$\begin{array}{lllllllllllllllll}\mathrm{Gem} / \mathrm{C} i \mathrm{~s} & 55 & 52 & 48 & 44 & 39 & 30 & 25 & 23 & 21 & 19 & 18 & 11 & 4 & 3 & 0 & 0\end{array}$

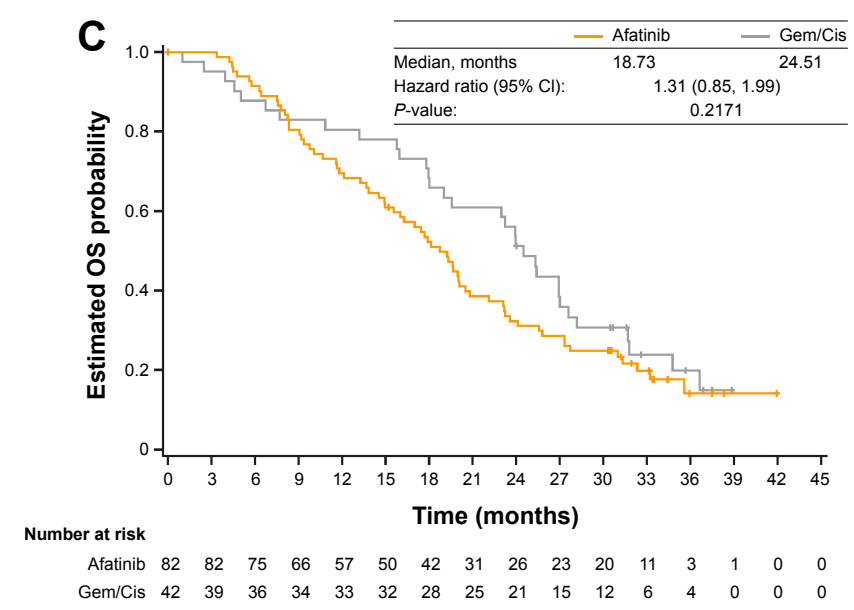

Figure 3 OS (A) in all Chinese patients in LUX-Lung 6, (B) in Chinese patients with Dell 9 mutations, and (C) in Chinese patients with the L858R mutation. Abbreviations: Cis, cisplatin; Gem, gemcitabine; OS, overall survival.

Table 2 Best overall tumor response by independent review in Chinese patients

\begin{tabular}{|c|c|c|c|}
\hline Parameter & $\begin{array}{l}\text { Afatinib } \\
(n=217)\end{array}$ & $\begin{array}{l}\text { Gem/Cis } \\
(n=110)\end{array}$ & $\begin{array}{l}\text { Afatinib vs } \mathrm{Gem} / \mathrm{Cis} \text { odds } \\
\text { ratio }(95 \% \mathrm{Cl})(P \text {-value })\end{array}$ \\
\hline Disease control, n (\%) & $200(92.2)$ & $85(77.3)$ & $3.45(I .77,6.7 I)(0.0003)$ \\
\hline Objective response & $145(66.8)$ & $26(23.6)$ & $6.94(4.05, \mathrm{II} .88)(<0.000 \mathrm{I})$ \\
\hline Complete response & $3(1.4)$ & 0 & - \\
\hline Partial response & $142(65.4)$ & $26(23.6)$ & - \\
\hline SD & 47 (2I.7) & $59(53.6)$ & - \\
\hline $\mathrm{NN}^{\mathrm{a}}$ & $8(3.7)$ & 0 & - \\
\hline PD & $8(3.7)$ & $6(5.5)$ & - \\
\hline $\mathrm{SD} / \mathrm{NN}$ for $<35$ days $^{\mathrm{b}}$ & 0 & $2(1.8)$ & - \\
\hline Not evaluable & $9(4.1)$ & $19(17.3)$ & - \\
\hline \multicolumn{4}{|c|}{ Objective response by mutation type, n/N (\%) } \\
\hline Dell9 & $86 / 111(77.5)$ & I5/55 (27.3) & $9.17(4.37,19.26)(<0.0001)$ \\
\hline L858R & $42 / 82(51.2)$ & $8 / 42(19.0)$ & $4.46(1.84,10.80)(0.0009)$ \\
\hline Others & $17 / 24(70.8)$ & $3 / 13(23.1)$ & $8.10(1.70,38.60)(0.0087)$ \\
\hline \multicolumn{4}{|c|}{ Disease control by mutation type, $\mathrm{n} / \mathrm{N}$ (\%) } \\
\hline Dell9 & 104/III (93.7) & $42 / 55(76.4)$ & $4.60(1.72,12.33)(0.0024)$ \\
\hline L858R & $72 / 82(87.8)$ & $33 / 42(78.6)$ & $1.96(0.73,5.29)(0.1817)$ \\
\hline Others & $24 / 24(100)$ & $10 / 13(76.9)$ & $\mathrm{NE}$ \\
\hline
\end{tabular}

Notes: astable nontarget disease in the absence of baseline target disease. ${ }^{b S D} / \mathrm{NN}$ best response but $<35$ days from randomization, followed by PD.

Abbreviations: Cis, cisplatin; CR, complete response; Gem, gemcitabine; NE, not evaluable; NN, non-CR/non-PD; PD, progressive disease; SD, stable disease. 
Table 3 Treatment-related AEs occurring in $>10 \%$ of Chinese patients in either treatment group

\begin{tabular}{|c|c|c|c|c|c|c|}
\hline \multirow[t]{2}{*}{ Patients with AEs, n (\%) } & \multicolumn{3}{|c|}{ Afatinib $(n=2 \mid 4)^{a}$} & \multicolumn{3}{|c|}{ Gem/Cis $(n=103)^{a}$} \\
\hline & All & Grade 3 & Grade 4 & All & Grade 3 & Grade 4 \\
\hline Any related $\mathrm{AE}$ & $211(98.6)$ & $76(35.5)$ & $2(0.9)$ & $102(99.0)$ & $35(35.9)$ & $22(21.4)$ \\
\hline Diarrhea & $187(87.4)$ & $12(5.6)$ & 0 & $10(9.7)$ & 0 & 0 \\
\hline Rash/acne ${ }^{b}$ & $173(80.8)$ & $34(15.9)$ & $\mathrm{I}(0.5)$ & $10(9.7)$ & 0 & 0 \\
\hline Stomatitis ${ }^{\mathrm{b}}$ & $105(49.1)$ & $13(6.1)$ & 0 & $3(2.9)$ & 0 & 0 \\
\hline Vomiting & $19(8.9)$ & $2(0.9)$ & 0 & $85(82.5)$ & $15(14.6)$ & $4(3.9)$ \\
\hline Nausea & $16(7.5)$ & 0 & 0 & $82(79.6)$ & $8(7.8)$ & $\mathrm{I}(\mathrm{I} .0)$ \\
\hline Neutropenia & $6(2.8)$ & $\mathrm{I}(0.5)$ & 0 & $60(58.3)$ & $19(18.4)$ & $10(9.7)$ \\
\hline Leukopenia & $8(3.7)$ & $\mathrm{I}(0.5)$ & 0 & $58(56.3)$ & $15(14.6)$ & $2(1.9)$ \\
\hline Nail effect ${ }^{\mathrm{b}}$ & $65(30.4)$ & 0 & 0 & 0 & 0 & 0 \\
\hline Decreased appetite & $21(9.8)$ & $2(0.9)$ & 0 & $43(4 \mid .7)$ & $\mathrm{I}(\mathrm{I} .0)$ & 0 \\
\hline ALT increased & $45(21.0)$ & $2(0.9)$ & 0 & $16(5.5)$ & $2(1.9)$ & I (I.0) \\
\hline Fatigue $^{\mathrm{b}}$ & $21(9.8)$ & 0 & 0 & 39 (37.9) & 0 & 0 \\
\hline AST increased & $35(16.4)$ & 0 & 0 & $10(9.7)$ & $2(1.9)$ & 0 \\
\hline Anemia & II (5.1) & $\mathrm{I}(0.5)$ & 0 & $28(27.2)$ & $8(7.8)$ & $2(1.9)$ \\
\hline Epistaxis & $31(14.5)$ & $\mathrm{I}(0.5)$ & 0 & I (I.0) & 0 & 0 \\
\hline White blood cell count decreased & $2(0.9)$ & 0 & 0 & $27(26.2)$ & $7(6.8)$ & 0 \\
\hline Neutrophil count decreased & $2(0.9)$ & 0 & 0 & $25(24.3)$ & $5(4.9)$ & $3(2.9)$ \\
\hline Hypokalemia & II (5.I) & $2(0.9)$ & 0 & $15(14.6)$ & $9(8.7)$ & 0 \\
\hline Hemoglobin decreased & $4(1.9)$ & $\mathrm{I}(0.5)$ & 0 & $20(19.4)$ & $3(2.9)$ & $\mathrm{I}(\mathrm{I} .0)$ \\
\hline Pruritus & $23(10.7)$ & 0 & 0 & 0 & 0 & 0 \\
\hline Thrombocytopenia & $2(0.9)$ & $\mathrm{I}(0.5)$ & 0 & $20(19.4)$ & $8(7.8)$ & $3(2.9)$ \\
\hline Constipation & $3(1.4)$ & 0 & 0 & $14(13.6)$ & 0 & 0 \\
\hline Alopecia & $6(2.8)$ & 0 & 0 & II (I0.7) & 0 & 0 \\
\hline Platelet count decreased & $2(0.9)$ & 0 & 0 & $12(11.7)$ & $3(2.9)$ & $2(1.9)$ \\
\hline
\end{tabular}

Notes: a Grade 5 AEs considered potentially related to treatment by the investigators were reported in one patient each treated with afatinib (sudden death) or gem/cis (cardiac failure). ${ }^{\mathrm{b}}$ Grouped term.

Abbreviations: AE, adverse event; ALT, alanine transaminase; AST, aspartate transaminase; Cis, cisplatin; Gem, gemcitabine.

respectively), but substantially more patients in the gemcitabine/ cisplatin group experienced grade 4 TRAEs (21.4\%) than in the afatinib group $(0.9 \%)$. The most common treatment-related grade $3 / 4$ AEs in the afatinib group were diarrhea $(5.6 \% / 0 \%)$, rash/acne $(16.4 \% / 0 \%)$, and stomatitis $(6.1 \% / 0 \%)$, whereas vomiting $(18.4 \% / 0.9 \%)$, nausea $(8.7 \% / 0 \%)$, neutropenia $(28.2 \% / 0.5 \%)$, and leukopenia $(16.5 \% / 0.5 \%)$ were the most common treatment-related grade $3 / 4$ AEs in the gemcitabine/ cisplatin group (Table 3 ).

TRAEs leading to permanent discontinuation of the study drug were reported in 12 patients $(5.6 \%)$ in the afatinib arm and 43 patients (41.7\%) in the gemcitabine/ cisplatin arm. No patients in the afatinib group discontinued treatment because of diarrhea; the most common reason for discontinuation was rash (5/12 patients). The most common reasons for discontinuing gemcitabine/cisplatin were vomiting $(15 / 43 ; 35 \%)$, nausea $(11 / 43 ; 26 \%)$, and neutropenia $(10 / 43 ; 23 \%)$.

Serious TRAEs were reported in $6.1 \%$ of patients in the afatinib group and $4.9 \%$ of patients in the gemcitabine/ cisplatin group. Two grade 5 (fatal) AEs were considered possibly related to treatment, one in each treatment group.
These were sudden death in the afatinib group and cardiac failure in the gemcitabine/cisplatin group.

\section{PROs}

Analysis of PROs focused on three key symptoms that are most important to patients with NSCLC: cough, dyspnea, and pain. Significantly greater proportions of patients in the afatinib group had improvements in cough $(76.8 \%$ versus $55.4 \%$; $P=0.0003)$, dyspnea $(71.3 \%$ versus $47.8 \%$; $P=0.0001)$, and pain $(63.9 \%$ versus $43.5 \% ; P=0.0012)$ than in the gemcitabine/cisplatin group (Figure 4). There were significant improvements in all items related to cough, dyspnea, and pain except "dyspnea rested" and "pain in arm/shoulder". Time to deterioration of cough (HR, 0.43 [95\% CI, 0.28, 0.67]; $P=0.0001$ ), dyspnea (HR, 0.51 [95\% CI, $0.37,0.71] ; P<0.0001$ ), and pain (HR, 0.62 [95\% CI, 0.45, 0.87 ; $P=0.0044)$ were all delayed with afatinib versus chemotherapy (Supplementary slides).

\section{Pharmacokinetics}

Afatinib trough plasma concentrations showed high variability (geometric coefficient of variation [ $\mathrm{gCV}] 47.1 \%-90.3 \%$ ) 


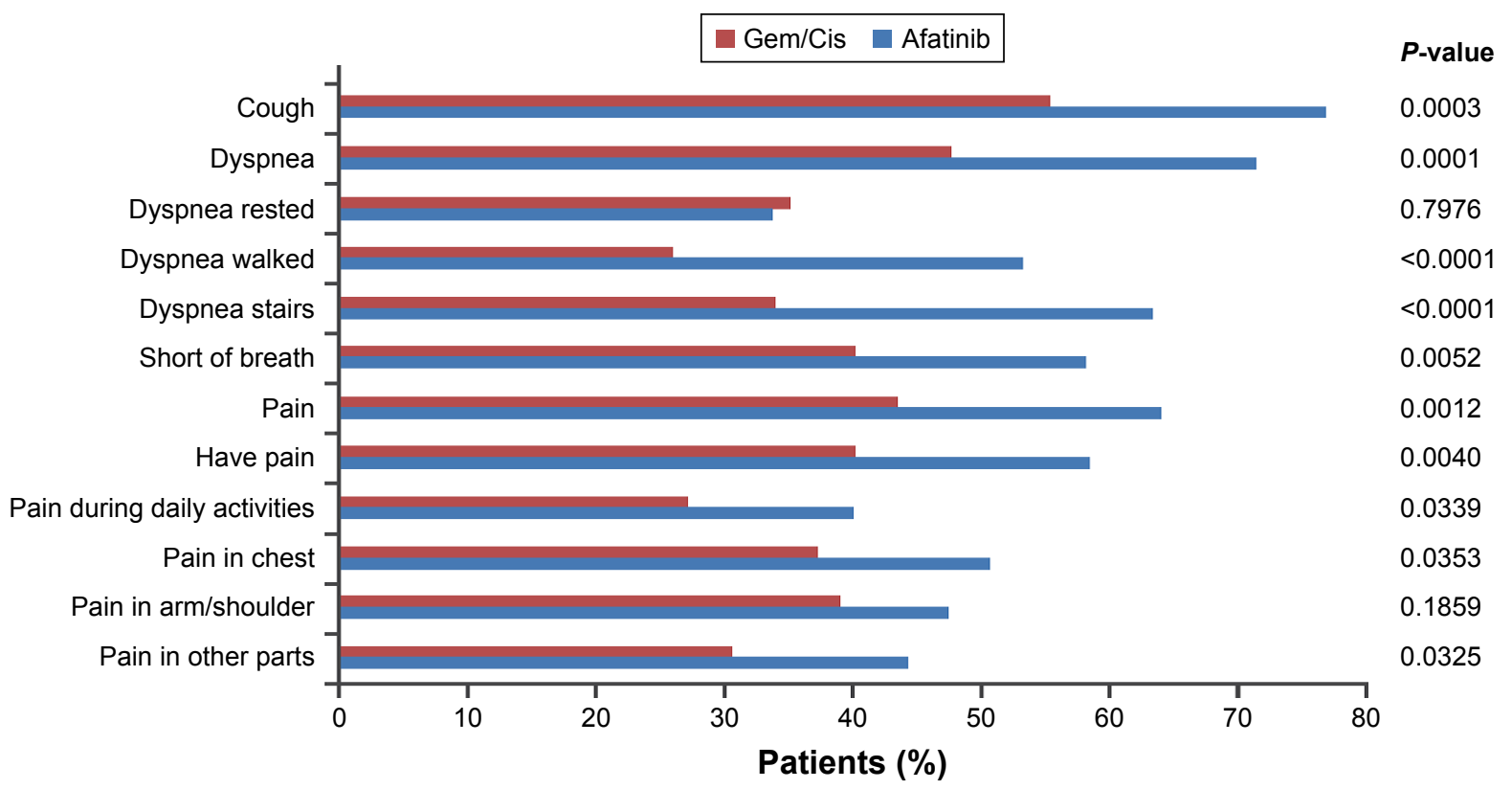

Figure 4 Percentage of patients who had improvements in cough, dyspnea, and pain. Outcomes assessed according to the European Organisation for Research and Treatment of Cancer Quality of Life Questionnaire C30 (QLQ-C30) and its lung cancer-specific module (QLQ-LCI3).

Abbreviations: $\mathrm{Cis}$, cisplatin; Gem, gemcitabine.

for all dose groups and over all pharmacokinetic (PK) visits (Table 4). Mean trough plasma concentrations at the last PK observation on day 43 , however, were at a similar level for all dose groups, by which time most patients would have received any required dose adjustments (Table 4 ). The geometric mean trough plasma concentration was $20.6 \mathrm{ng} / \mathrm{mL}$ (gCV $47.1 \%$ ) for patients whose dose had been reduced to afatinib $30 \mathrm{mg}, 21.5 \mathrm{ng} / \mathrm{mL}$ (gCV 63.5\%) for patients who remained on afatinib $40 \mathrm{mg}$, and $23.5 \mathrm{ng} / \mathrm{mL}$ (gCV 61.5\%) for patients whose dose had been escalated to afatinib $50 \mathrm{mg}$.

\section{Discussion}

To our knowledge, this subgroup analysis of LUX-Lung 6 is the largest analysis of treatment with an EGFR-targeted agent, following upfront mutation testing, in Chinese patients. Our analysis demonstrated that afatinib significantly improved PFS, ORR, and (in patients with an EGFR Del19 mutation) OS versus gemcitabine/cisplatin in patients from mainland China. Furthermore, efficacy benefits were largely consistent across patient subgroups, including mutation type (Del19 or L858R). Importantly, the efficacy benefits with afatinib were complemented by improvements in PROs for symptoms that are most important to patients with NSCLC: cough, dyspnea, and pain. There were 20 LTRs, of whom eight remain on treatment at the time of analysis. Overall, the tolerability profile of afatinib was consistent with previous experience and was considerably less severe than that experienced with platinum-based chemotherapy; TRAEs were largely manageable on treatment and discontinuation due to AEs was rare. Together, these data demonstrate that afatinib should be considered as a first-line treatment option in Chinese patients with EGFR mutation-positive NSCLC.

Given the availability of several EGFR TKIs in China, it is a pertinent question to ask the following: which agent is the most suitable first-line treatment of choice in which patient? At present, few head-to-head clinical trial data comparing EGFR TKIs in Chinese patients are available to help guide treatment decisions. The recent CTONG0901 Phase III trial

Table 4 Geometric mean trough plasma concentrations of afatinib after multiple daily dosing of afatinib $40 \mathrm{mg}$ and after dose escalation to afatinib $50 \mathrm{mg}$ or dose reduction to afatinib $30 \mathrm{mg}$

\begin{tabular}{|c|c|c|c|c|c|c|c|c|c|}
\hline \multirow[t]{2}{*}{ Visit } & \multicolumn{3}{|c|}{ Afatinib $30 \mathrm{mg}$} & \multicolumn{3}{|c|}{ Afatinib $40 \mathrm{mg}$} & \multicolumn{3}{|c|}{ Afatinib $50 \mathrm{mg}$} \\
\hline & $\mathbf{N}$ & $\begin{array}{l}\text { gMean, } \\
\text { ng/mL }\end{array}$ & gCV, \% & $\mathbf{N}$ & $\begin{array}{l}\text { gMean, } \\
\mathrm{ng} / \mathrm{mL}\end{array}$ & $\mathrm{gCV}, \%$ & $\mathbf{N}$ & $\begin{array}{l}\text { gMean, } \\
\text { ng/mL }\end{array}$ & gCV, \% \\
\hline Course 2 visit I (day 22) & 0 & NE & $\mathrm{NE}$ & 187 & 22.6 & 60.9 & 0 & NE & NE \\
\hline Course 2 visit 2 (day 29) & 6 & 19.5 & 90.3 & 143 & 23.5 & 63.0 & 33 & 23.3 & 60.6 \\
\hline Course 3 visit I (day 43) & 17 & 20.6 & 47.1 & 140 & 21.5 & 63.5 & 34 & 23.5 & 61.5 \\
\hline
\end{tabular}

Abbreviations: gCV, geometric coefficient of variation; gMean, geometric mean; NE, not evaluable. 
compared the efficacy and safety of gefitinib and erlotinib in 265 Chinese patients with EGFR mutation-positive NSCLC in any line. Findings showed no significant difference in PFS (median 13.0 versus 10.4 months; HR, 0.81 [95\% CI, 0.62, 1.05]; $P=0.108$ ) or OS (median 22.9 versus 20.1 months; HR, 0.84 [95\% CI, 0.63, 1.13]; $P=0.250$ ) between the two treatment arms. ${ }^{26}$ LUX-Lung 7 is the only published headto-head trial between two EGFR-targeted agents specifically in a first-line setting. In this trial, 48 of 319 randomized patients were from China. For the overall dataset, afatinib significantly improved PFS (median 11.0 versus 10.9 months; HR, 0.73 [95\% CI, 0.57, 0.95]; $P=0.017$ ) and TTF (median 13.7 versus 11.5 months; HR, 0.73 [95\% CI, 0.58, 0.92]; $P=0.0073$ ) with a trend toward improved OS, despite not being powered to detect any difference in OS versus gefitinib (median OS 27.9 versus 24.5 months; HR, 0.85 [95\% CI, $0.66,1.09] ; P=0.1950) .{ }^{17,18,27}$

Although cross-trial comparisons are difficult, and clearly do not substitute for prospective head-to-head data, it is of interest to view this large LUX-Lung 6 subanalysis $(n=327)$ dataset in the context of available Chinese data with erlotinib, gefitinib, and icotinib. Two trials provide data on erlotinib in Chinese patients: the Phase III OPTIMAL trial examined the efficacy and safety of erlotinib $(n=82)$ versus gemcitabine/ carboplatin $(\mathrm{n}=72)$ in Chinese patients with EGFR mutationpositive NSCLC (common mutations only), ${ }^{14,28}$ while a subsequent Asian Phase III trial, ENSURE - which largely included Chinese patients $(\sim 80 \%)$ - compared erlotinib $(n=110)$ versus gemcitabine/cisplatin $(\mathrm{n}=107)$ in the same treatment setting. ${ }^{13}$ The recent Phase III CONVINCE trial compared icotinib $(n=148)$ versus cisplatin/pemetrexed $(n=137)$ in Chinese patients with EGFR mutation-positive NSCLC (common mutations only). ${ }^{15}$ In contrast to erlotinib and icotinib, few data are available that have assessed gefitinib specifically in Chinese patients with EGFR mutation-positive NSCLC. The Phase III trials, NEJ-002 and WJTOG3405, were undertaken specifically in Japanese patients. ${ }^{7,8}$ Although IPASS included 372 patients from China, analysis in EGFR mutation-positive patients was difficult to interpret as a low proportion of patients recruited in China provided a tumor sample to determine EGFR mutation status (38\%). ${ }^{29}$ In OPTIMAL, erlotinib conferred PFS advantage versus chemotherapy in Chinese patients (HR, 0.16 [95\% CI, 0.10, 0.26]; $P<0.0001$ ), although it should be noted that efficacy was analyzed in patients who "had received at least one dose of study drug" rather than in the intent-to-treat population. Additionally, no clinical study report was available for this trial when reviewed by the European Medicines Agency. PFS advantage with erlotinib was also observed in a subgroup analysis of Chinese patients in the ENSURE study, but with a higher HR than that is observed in OPTIMAL (HR, 0.30 [95\% CI, 0.18, 0.49]). ${ }^{13,14}$ In the Chinese subanalysis of IPASS, there was no significant difference in PFS with gefitinib and chemotherapy in EGFR mutation unselected patients (median 6.8 versus 6.8 months; HR, 0.79 [95\% CI, 0.62, 1.01]; $P=0.065) .{ }^{29} \mathrm{In}$ CONVINCE, icotinib significantly improved PFS versus chemotherapy in Chinese patients (median 11.2 versus 7.9 months; HR, 0.61 [95\% CI, 0.43, 0.87]; $P=0.006) .{ }^{15}$

Like afatinib, gefitinib, icotinib, and erlotinib were associated with better tolerability profiles compared to chemotherapy in Chinese patients with NSCLC. ${ }^{13-15,29}$ TRAEs were reported in $87.0 \%-87.3 \%$ with erlotinib, $83.2 \%$ with gefitinib, and $54.1 \%$ with icotinib. Treatment-related serious AEs were reported in $2.4 \%-2.7 \%$ with erlotinib and $2.2 \%$ with gefitinib. The most common grade $3 / 4$ AEs with erlotinib were rash $(2.4 \%-6.4 \%)$ and elevated alanine transaminase (ALT; 3.6\% in OPTIMAL; none reported in ENSURE); the most common grade 3/4 AEs with gefitinib were rash/ acne (3.8\%) and elevated ALT (3.8\%). The most common grade $3 / 4$ AEs with icotinib were rash (14.9\%) and diarrhea (7.4\%). Treatment discontinuations due to AEs were required in $1.2 \%-2.7 \%$ of patients treated with erlotinib and $2.0 \%$ of patients treated with icotinib; no dose discontinuations because of AEs were required with gefitinib in the Chinese subanalysis of IPASS.

One important distinguishing feature of the LUX-Lung 6 Chinese data compared with available Chinese data for gefitinib, icotinib, and erlotinib is that afatinib uniquely conferred OS advantage versus chemotherapy in patients with an $E G F R$ Del19 mutation. This finding is consistent with the overall LUX-Lung 6 dataset of Del19 patients (median 31.4 versus 18.4 months; HR, 0.64 [95\% CI, 0.44, 0.94]), as well as the LUX-Lung 3 study (median 33.3 versus 21.1 months; HR, 0.54 [95\% CI, 0.36, 0.79]). ${ }^{16}$ In contrast, erlotinib did not confer OS advantage over chemotherapy in Del19 patients in either OPTIMAL (HR, 1.52 [95\% CI, 0.91, 2.52]) or ENSURE (HR, 0.79 [95\% CI, 0.48, 1.30]). ${ }^{13,28}$ Likewise, no OS advantage was observed with icotinib versus chemotherapy in Del19 patients in CONVINCE. ${ }^{15}$ The observation of OS benefit with afatinib in patients with Del19 mutations is unlikely to be attributable to imbalances in post-progression therapy because crossover rates in LUX-Lung 6 were similar in patients with Del19 and L858R mutations. ${ }^{16}$ It is possible that the achievement of significant OS benefit with afatinib but not erlotinib nor icotinib reflects the differences in mechanism of action between afatinib and first-generation 
TKIs. Notably, afatinib potently and irreversibly inhibits all members of the ErbB family, whereas first-generation TKIs reversibly inhibit EGFR only. ${ }^{30,31}$

In this subanalysis of LUX-Lung 6, TRAEs were reported by most patients, and treatment with afatinib was associated with the expected gastrointestinal and dermatologic AEs. These were managed with supportive care and protocol-defined dose reductions; post hoc analyses demonstrated that dose reduction effectively mitigated TRAEs without impacting efficacy. Consequently, few patients had to discontinue afatinib treatment because of AEs (5.6\%), and none because of diarrhea. This suggests that the established protocols for managing AEs associated with afatinib are robust and effective in Chinese patients. Furthermore, although AEs were common with afatinib, substantial improvements in health-related quality of life were reported, including improvements in all domains of lung-cancer symptoms, suggesting that the beneficial effects of afatinib outweigh its AEs.

Consistent with previous studies, the variability in afatinib plasma concentrations was high for all dose groups. Analysis of the LUX-Lung 3 and overall LUX-Lung 6 data has demonstrated that, in general, patients with high trough plasma concentrations were more likely to require a dose reduction to afatinib $30 \mathrm{mg}$ within the PK observation period of 43 days. ${ }^{19}$ The PK data suggest that the individual tolerability-driven dose modification is effective at reducing excessive afatinib levels. Importantly, observations from the overall LUX-Lung 3 and LUX-Lung 6 datasets showed that such tolerability-guided dose adjustment does not affect therapeutic efficacy. In both cases, median PFS was similar in patients who reduced their dose during the first 6 months versus those who did not (LUX-Lung 3: 11.3 versus 11.0 months; HR, 1.25 [95\% CI, 0.91, 1.72]; LUX-Lung 6: 12.3 versus 10.0 months; HR, 1.00 [95\% CI, 0.69, 1.46]). ${ }^{19}$

Limitations of this analysis include that it is a subgroup analysis of a larger study; however, it is notable that the population of over 300 Chinese patients represented $\sim 90 \%$ of the population of the parent study. ${ }^{12}$ The open-label nature of the study is also a limitation, although, in this analysis, assessment of the patients' scans was conducted by an independent central panel of radiologists and oncologists, and sensitivity analyses did not suggest any bias. Finally, it should be noted that chemotherapy would not now be regarded as the stateof-the-art comparator arm in randomized trials in patients with EGFR mutation-positive NSCLC; since the initiation of LUX-Lung 6 in 2010, TKIs have become the first-line treatment of choice in this setting. However, gemcitabine/ cisplatin was commonly used in Asia at the time that the study was designed, and the regulatory authorities approved this choice as a comparator. ${ }^{12}$

\section{Conclusion}

This analysis of Chinese patients with EGFR mutationpositive NSCLC has demonstrated that afatinib is more effective and has more durable effects than standard first-line chemotherapy. Furthermore, to our knowledge, afatinib is the only TKI to demonstrate improved OS versus standard chemotherapy in patients harboring an EGFR Del19 mutation. Together, the findings suggest that afatinib should be considered a first-line treatment option for this patient population.

\section{Acknowledgments}

We thank the patients, their families, and all the investigators who participated in this study. The authors were fully responsible for all content and editorial decisions, were involved at all stages of manuscript development, and have approved the final version. This work was supported by Boehringer Ingelheim. Medical writing assistance, supported financially by Boehringer Ingelheim, was provided by Lynn Pritchard of GeoMed, an Ashfield company, part of UDG Healthcare plc, during the preparation of this manuscript.

\section{Disclosure}

Y-LW reports consultancy fees from AstraZeneca; and lecture fees from AstraZeneca, Eli Lilly, Pfizer, Roche, and Sanofi. CZ reports lecture fees from AstraZeneca, Boehringer Ingelheim, Eli Lilly, Roche, and Sanofi. AM, JF, and BP report employment by Boehringer Ingelheim. The authors report no other conflicts of interest in this work.

\section{References}

1. Chen W, Zheng R, Zeng H, Zhang S. The incidence and mortality of major cancers in China, 2012. Chin J Cancer. 2016;35(1):73.

2. Hong QY, Wu GM, Qian GS, et al; Lung Cancer Group of Chinese Thoracic Society; Chinese Alliance Against Lung Cancer. Prevention and management of lung cancer in China. Cancer. 2015;121(Suppl 17) 3080-3088.

3. Li MJ, He Q, Li M, Luo F, Guan YS. Role of gefitinib in the targeted treatment of non-small-cell lung cancer in Chinese patients. Onco Targets Ther. 2016;9(9):1291-1302.

4. Reck M, Heigener DF, Mok T, Soria JC, Rabe KF. Management of nonsmall-cell lung cancer: recent developments. Lancet. 2013;382(9893): 709-719.

5. Reck M, Popat S, Reinmuth N, et al. ESMO Guidelines Working Group. Metastatic non-small-cell lung cancer (NSCLC): ESMO clinical practice guidelines for diagnosis, treatment and follow-up. Ann Oncol. 2014;25(Suppl 3):iii27-iii39.

6. Shi Y, Sun Y, Ding C, et al. China experts consensus on icotinib for non-small cell lung cancer treatment (2015 version). J Thorac Dis. 2015; 7(10):E468-E472. 
7. Maemondo M, Inoue A, Kobayashi K, et al; North-East Japan Study Group. Gefitinib or chemotherapy for non-small-cell lung cancer with mutated EGFR. N Engl J Med. 2010;362(25):2380-2388.

8. Mitsudomi T, Morita S, Yatabe Y, et al; West Japan Oncology Group. Gefitinib versus cisplatin plus docetaxel in patients with non-smallcell lung cancer harbouring mutations of the epidermal growth factor receptor (WJTOG3405): an open label, randomised phase 3 trial. Lancet Oncol. 2010;11(2):121-128.

9. Mok TS, Wu YL, Thongprasert S, et al. Gefitinib or carboplatinpaclitaxel in pulmonary adenocarcinoma. N Engl J Med. 2009;361(10): 947-957.

10. Rosell R, Carcereny E, Gervais R, et al; Spanish Lung Cancer Group in collaboration with Groupe Français de Pneumo-Cancérologie and Associazione Italiana Oncologia Toracica. Erlotinib versus standard chemotherapy as first-line treatment for European patients with advanced EGFR mutation-positive non-small-cell lung cancer (EURTAC): a multicentre, open-label, randomised phase 3 trial. Lancet Oncol. 2012;13(3):239-246.

11. Sequist LV, Yang JC, Yamamoto N, et al. Phase III study of afatinib or cisplatin plus pemetrexed in patients with metastatic lung adenocarcinoma with EGFR mutations. J Clin Oncol. 2013;31(27):3327-3334.

12. Wu YL, Zhou C, Hu CP, et al. Afatinib versus cisplatin plus gemcitabine for first-line treatment of Asian patients with advanced non-small-cell lung cancer harbouring EGFR mutations (LUX-Lung 6): an open-label, randomised phase 3 trial. Lancet Oncol. 2014;15(2):213-222.

13. Wu YL, Zhou C, Liam CK, et al. First-line erlotinib versus gemcitabine/ cisplatin in patients with advanced EGFR mutation-positive non-smallcell lung cancer: analyses from the phase III, randomized, open-label, ENSURE study. Ann Oncol. 2015;26(9):1883-1889.

14. Zhou C, Wu YL, Chen G, et al. Erlotinib versus chemotherapy as first-line treatment for patients with advanced EGFR mutation-positive non-small-cell lung cancer (OPTIMAL, CTONG-0802): a multicentre, open-label, randomised, phase 3 study. Lancet Oncol. 2011;12(8): $735-742$.

15. Shi YK, Wang L, Han BH, et al. First-line icotinib versus cisplatin/ pemetrexed plus pemetrexed maintenance therapy for patients with advanced EGFR mutation-positive lung adenocarcinoma (CONVINCE): a phase 3, open-label, randomized study. Ann Oncol. 2017;28(10): 2443-2450.

16. Yang JC, Wu YL, Schuler M, et al. Afatinib versus cisplatin-based chemotherapy for EGFR mutation-positive lung adenocarcinoma (LUXLung 3 and LUX-Lung 6): analysis of overall survival data from two randomised, phase 3 trials. Lancet Oncol. 2015;16(2):141-151.

17. Park K, Tan EH, O'Byrne K, et al. Afatinib versus gefitinib as first-line treatment of patients with EGFR mutation-positive non-small-cell lung cancer (LUX-Lung 7): a phase 2B, open-label, randomised controlled trial. Lancet Oncol. 2016;17(5):577-589.

18. Paz-Ares L, Tan EH, O’Byrne K, et al. Afatinib versus gefitinib in patients with EGFR mutation-positive advanced non-small-cell lung cancer: overall survival data from the phase IIb LUX-Lung 7 trial. Ann Oncol. 2017;28(2):270-277.
19. Yang JC, Sequist LV, Zhou C, et al. Effect of dose adjustment on the safety and efficacy of afatinib for EGFR mutation-positive lung adenocarcinoma: post hoc analyses of the randomized LUX-Lung 3 and 6 trials. Ann Oncol. 2016;27(11):2103-2110.

20. Schuette W, Schirmacher P, Eberhardt WE, et al. EGFR mutation status and first-line treatment in patients with stage III/IV non-small cell lung cancer in Germany: an observational study. Cancer Epidemiol Biomarkers Prev. 2015;24(8):1254-1261.

21. Sekine I, Yamamoto N, Nishio K, Saijo N. Emerging ethnic differences in lung cancer therapy. Br J Cancer. 2008;99(11):1757-1762.

22. Skov BG, Hogdall E, Clementsen P, et al. The prevalence of EGFR mutations in non-small cell lung cancer in an unselected Caucasian population. APMIS. 2015;123(2):108-115.

23. Shi Y, Li J, Zhang S, et al. Molecular epidemiology of EGFR mutations in Asian patients with advanced non-small-cell lung cancer of adenocarcinoma histology - Mainland China subset analysis of the PIONEER study. PLoS One. 2015;10(11):e0143515.

24. Aaronson NK, Ahmedzai S, Bergman B, et al. The European Organization for Research and treatment of cancer QLQ-C30: a quality-of-life instrument for use in international clinical trials in oncology. $J$ Natl Cancer Inst. 1993;85(5):365-376.

25. Bergman B, Aaronson NK, Ahmedzai S, Kaasa S, Sullivan M. The EORTC QLQ-LC13: a modular supplement to the EORTC Core Quality of Life Questionnaire (QLQ-C30) for use in lung cancer clinical trials. EORTC Study Group on quality of life. Eur J Cancer. 1994;30A(5): 635-642.

26. Yang JJ, Zhou Q, Yan HH, et al. A phase III randomised controlled trial of erlotinib vs gefitinib in advanced non-small cell lung cancer with EGFR mutations. Br J Cancer. 2017;116(5):568-574.

27. Corral J, Park K, Yang JCH, et al. Afatinib (A) vs gefitinib (G) in patients with EGFR mutation-positive (EGFRm+) NSCLC: updated OS data from the phase IIb trial LUX-Lung 7 (LL7). Poster presented at: European Lung Cancer Conference; May 07, 2017; Geneva, Switzerland.

28. Zhou C, Wu YL, Chen G, et al. Final overall survival results from a randomised, Phase III study of erlotinib versus chemotherapy as firstline treatment of EGFR mutation-positive advanced non-small-cell lung cancer (OPTIMAL, CTONG-0802). Ann Oncol. 2015;26(9): 1877-1883.

29. Wu YL, Chu DT, Han B, et al. Phase III, randomized, open-label, firstline study in Asia of gefitinib versus carboplatin/paclitaxel in clinically selected patients with advanced non-small-cell lung cancer: evaluation of patients recruited from mainland China. Asia Pac J Clin Oncol. 2012; $8(3): 232-243$.

30. Li D, Ambrogio L, Shimamura T, et al. BIBW2992, an irreversible EGFR/HER2 inhibitor highly effective in preclinical lung cancer models. Oncogene. 2008;27(34):4702-4711.

31. Solca F, Dahl G, Zoephel A, et al. Target binding properties and cellular activity of afatinib (BIBW 2992), an irreversible ErbB family blocker. J Pharmacol Exp Ther. 2012;343(2):342-350. 
Video abstract

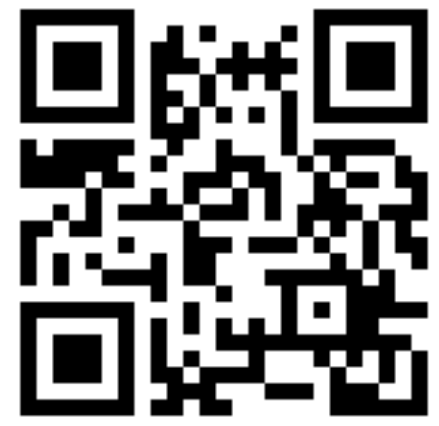

Point your SmartPhone at the code above. If you have a QR code reader the video abstract will appear. Or use: http://youtu.be/llG80m9qlkc

\section{Publish your work in this journal}

OncoTargets and Therapy is an international, peer-reviewed, open access journal focusing on the pathological basis of all cancers, potential targets for therapy and treatment protocols employed to improve the management of cancer patients. The journal also focuses on the impact of management programs and new therapeutic agents and protocols on patient perspectives such as quality of life, adherence and satisfaction. The manuscript management system is completely online and includes a very quick and fair peer-review system, which is all easy to use. Visit http://www.dovepress.com/testimonials.php to read real quotes from published authors. 